\title{
A Simple Geometrical Path Towards Hybrid Orbitals
}

\author{
Denis Rafael Nacbar ${ }^{a, b}$, Allan Victor Ribeiro ${ }^{b, c *}$, Alexys Bruno-Alfonso $^{d}$ \\ aInstituto Federal de Educação, Ciência e Tecnologia de São Paulo - IFSP, CEP 12929-600, \\ Bragança Paulista, SP, Brazil \\ ${ }^{\text {b} P r o g r a m a ~ d e ~ P o ́ s-G r a d u a c ̧ a ̃ o ~ e m ~ C i e ̂ n c i a ~ e ~ T e c n o l o g i a ~ d e ~ M a t e r i a i s ~-~ P O S M A T, ~}$ \\ Universidade Estadual Paulista - Unesp, CEP 17033-360, Bauru, SP, Brazil \\ 'Instituto Federal de Educação, Ciência e Tecnologia de São Paulo - IFSP, CEP 16201-407, \\ Birigui, SP, Brazil \\ ${ }^{d}$ Departamento de Matemática, Faculdade de Ciências, Universidade Estadual Paulista - Unesp, \\ CEP 17033-360, Bauru, SP, Brazil
}

Received: April 18, 2014; Revised: September 10, 2014

\begin{abstract}
It is shown that the standard $s p^{n}$ hybrid orbitals are orthogonal orbitals that minimize the total quadratic spread. This is done in a concise way that may improve the understanding of hybrid orbitals. The fact that maximally localized Wannier functions of crystalline materials may resemble hybrid orbitals is discussed.
\end{abstract}

Keywords: hybrid orbitals, localization, Wannier functions

\section{Introduction}

Hybrid atomic orbitals were introduced, in 1931, by the Nobel Prize winner Linus Pauling, in order to explain the nature of the chemical bond on quantum mechanical grounds ${ }^{1}$. Since then, these orbitals have been successfully used to describe single and multiple bonds between atoms ${ }^{2,3}$. Although a thorough understanding of the hybrid orbitals rely on energy calculations, geometrical considerations ${ }^{4}$, the maximization of the overlap integrals ${ }^{5}$, and group-theoretical approaches $^{6}$ have been proposed.

The aim of this work is to present an alternative approach motivated by modern methods of Materials Science involving Wannier functions ${ }^{7}$. Such functions were introduced in solid-state physics by G. H. Wannier, in 1937. They form a complete basis of the space of electronic states of a crystal, just like the Bloch functions do. However, the former ones are localized, in contrast with the extended character of the latter. The Wannier functions lack unicity and some effort has been devoted to the development of analytical and numerical techniques that optimize their degree of localization ${ }^{7}$. We show that the hybrid $s p^{n}$ orbitals are the most localized among the orthogonal linear combinations of one $\mathrm{s}$ and $n \mathrm{p}$ orbitals. This is helpful to understand why maximally localized Wannier functions may resemble the hybrid orbitals $\mathrm{s}^{7,8}$.

A similar path towards the hybrid orbitals has been used in previous works. In 1952, Hurley ${ }^{9}$ addressed this problem in his Doctoral Thesis. A decade later, Boys ${ }^{10,11}$ used the same localization criterion, while Edmiston and Ruedenberg ${ }^{12,13}$ dealt with the minimization of the electrostatic energy. Since the mentioned works are hardly available or give no mathematical details on the hybrid orbitals, we present an independent approach. In Section 2, we derive the hybrid

*e-mail: allan_vr@fc.unesp.br orbitals as maximally localized states, according to the Foster-Boys criterion. The concluding remarks are given in Section 3.

\section{Minimization of the Total Quadratic Spread}

The degree of localization of a wave function may be expressed by the variance of the associated probability distribution ${ }^{7}$. In this sense, a state is more localized if its distribution has less variance. The variance of an electronic state $\psi$ is given by:

$\sigma^{2}(\psi)=\left\langle\psi\left|\boldsymbol{r}^{2}\right| \psi\right\rangle-\langle\psi|\boldsymbol{r}| \psi\rangle^{2}$

where $r=\left(x_{1}, x_{2}, x_{3}\right)$ is the particle position.

Let us consider an orthonormal set of states $\phi_{0}, \phi_{1}, \ldots, \phi_{n}$. These states can be linearly combined to produce a new set of orthonormal states $\psi_{0}, \psi_{1}, \ldots, \psi_{n}$, i.e.,

$\psi_{j}=\sum_{k=0}^{n} c_{j, k} \phi_{k}$

with $j=0, \ldots, n$. The coefficients of the linear combination fulfill

$$
\sum_{k=0}^{n} c_{j, k}^{*} c_{j^{\prime}, k}=\delta_{j, j^{\prime}}
$$

where $\delta_{j, j}$, is the Kronecker symbol. This is equivalent to

$\phi_{k}=\sum_{j=0}^{n} c_{j, k}^{*} \psi_{j}$

with

$$
\sum_{j=0}^{n} c_{j, k}^{*} c_{j, k^{\prime}}=\delta_{k, k^{\prime}}
$$


The total quadratic spread of each set of states is a Foster-Boys functional ${ }^{7}$. For the sets $\phi_{0}, \phi_{1}, \ldots, \phi_{n}$ and $\psi_{0}, \psi_{1}, \ldots, \psi_{n}$, the functional is given by

$\Omega(\phi)=\sum_{k=0}^{n} \sigma^{2}\left(\phi_{k}\right)$

and

$\Omega(\psi)=\sum_{j=0}^{n} \sigma^{2}\left(\psi_{j}\right)$

respectively. Taking Equation 5 into account, the difference between Equations 7 and 6 simplifies to

$\Delta \Omega=\Omega(\psi)-\Omega(\phi)=\sum_{k=0}^{n} \vec{r}_{k, k}^{2}-\sum_{j=0}^{n}\left(\sum_{k, k^{\prime}=0}^{n} c_{j, k}^{*} \vec{r}_{k, k^{\prime}} c_{j, k^{\prime}}\right)^{2}$

where

$$
\vec{r}_{k, k^{\prime}}=\left\langle\phi_{k}|\vec{r}| \phi_{k^{\prime}}\right\rangle
$$

When one considers the states $\phi_{0}, \phi_{1}, \ldots, \phi_{n}$ as atomic orbitals whose probability distribution presents inversion symmetry, the condition $\vec{r}_{k, k^{\prime}}=\overrightarrow{0}$ applies for $k, k^{\prime}=0, \ldots, n$. Hence,

$$
\Delta \Omega=-\sum_{j=0}^{n}\left(\sum_{\substack{k, k^{\prime}=0 \\ k \neq k^{\prime}}}^{n} c_{j, k}^{*} \vec{r}_{k, k^{\prime}} c_{j, k^{\prime}}\right)^{2}
$$

This should be minimized, taking into account the $n(n+1) / 2$ independent conditions given by Equation 5 .

To deal with the hybridization of one $\mathrm{s}$ and $n \mathrm{p}$ orbitals of a given atomic shell, we take $n=1, \ldots, 3$,

$\phi_{0}=\mathrm{s}=\frac{R_{0}(r)}{\sqrt{4 \pi}}$

and

$\phi_{k}=\mathrm{p}_{x_{k}}=\frac{R_{1}(r)}{\sqrt{\frac{4 \pi}{3}}} \frac{x_{k}}{r}$

for $k=1, \ldots, n . R_{0}(r)$ and $R_{1}(r)$ are the radial parts of the respective orbitals.

By taking into account the symmetries of orbitals in Equations 11 and 12, Equation 10 simplifies to

$\Delta \Omega=-4 X^{2} \sum_{j=0}^{n} \sum_{k=1}^{n} \operatorname{Re}^{2}\left(c_{j, 0}^{*} c_{j, k}\right)$

where

$X=\left\langle\mathrm{s}\left|x_{1}\right| \mathrm{p}_{x_{1}}\right\rangle$

From the physical point of view, no generality is lost by assuming

$c_{j, 0} \geq 0$

for every $j$. Hence, according to Equation 3, we obtain

$$
\begin{aligned}
\Delta \Omega= & -4 X^{2} \sum_{j=0}^{n}\left(c_{j, 0}^{2} \sum_{k=1}^{n} \operatorname{Re}^{2}\left(c_{j, k}\right)\right)=4 X^{2} \sum_{j=0}^{n} c_{j, 0}^{2}\left(c_{j, 0}^{2}-1\right)+ \\
& 4 X^{2} \sum_{j=0}^{n} \sum_{k=1}^{n} \operatorname{Im}^{2}\left(c_{j, k}\right)
\end{aligned}
$$

This will be minimized, satisfying the conditions in Equations 5 and 15.

The conditions in Equation 5 may be stated in terms of the vectors $\vec{u}_{k}=\left(c_{0, k}, \ldots, c_{n, k}\right)$, with $k=0, \ldots, n$. They form an orthonormal set in $\mathrm{C}^{n+1}$, where $\mathrm{C}$ denotes the set of complex numbers. In particular, for a given unit vector $\vec{u}_{0}$, it is required the existence of an orthonormal set of vectors $\vec{u}_{1}, \ldots, \vec{u}_{n}$ that are orthogonal to $\vec{u}_{0}$. Since $\vec{u}_{0}$ is real, this requirement is always fulfilled by a set of real vectors $\vec{u}_{1}, \ldots, \vec{u}_{n}$. This means that a set of real hybrid orbitals will minimize the total quadratic spread.

For real unit vectors $\vec{u}_{0}, \vec{u}_{1}, \ldots, \vec{u}_{n}$, Equation 16 takes the form

$\Delta \Omega=4 X^{2}(f-1)$

with

$f=\sum_{j=0}^{n} c_{j, 0}^{4}$

The minimization of $\Delta \Omega$ should be performed under the restrictions (Equation 15) and $G=1$, where

$G=\sum_{j=0}^{n} c_{j, 0}^{2}$

The global minimum of $f$ under the condition (Equation 19) may be found by using the method of Lagrange multipliers. The existence of the global minimum is guaranteed by the fact that the objective function is continuous and the restrictions lead to a closed limited subset of $\mathrm{R}^{n+1}$, where $\mathrm{R}$ is the set of real numbers ${ }^{14}$. The values of $c_{0,0}, \ldots, c_{n, 0}$ must satisfy the equations

$\frac{\partial f}{\partial c_{j, 0}}=\lambda \frac{\partial G}{\partial c_{j, 0}}$

for $j=0, \ldots, n$, where $\lambda$ is the Lagrange multiplier. After computing the partial derivatives, Equation 20 simplifies to

$\left(c_{j, 0}^{2}-\frac{\lambda}{2}\right) c_{j, 0}=0$

The system of equations in Equation 21 has $2^{n+1}-1$ meaningful solutions. In fact, each $c_{j, 0}$ is either 0 or $\sqrt{\lambda / 2}$, and at least one of the coefficients $c_{j, 0}$ is not zero. The solutions may be classified in terms of the number $m+1$ of non-null coefficients $c_{j, 0}$, with $m=0, \ldots, n$. Since $G=1$, we obtain $c_{j, 0}=\frac{1}{\sqrt{m+1}}$, for $m+1$ values of $j$, and $c_{j, 0}=0$ for the other $n-m$ values of $j$. For each $m$, the value of $f$ is given by $f_{m}=1 /(m+1)$, and the global minimum of $f$ corresponds to $m=n$. Therefore, the minimum value of the total quadratic spread corresponds to

$\Delta \Omega_{n}=-\frac{4 n X^{2}}{n+1}$

namely, $\Delta \Omega_{1}=-2 X^{2}, \Delta \Omega_{2}=-8 X^{2} / 3$, and $\Delta \Omega_{3}=-3 X^{2}$.

To deal with the shape of the states $\psi_{0}, \psi_{1}, \ldots, \psi_{n}$, we introduce the vectors $v_{j}=\left(c_{j, 1}, \ldots, c_{j, n}\right)$. From Equation 3, we obtain

$\left|\boldsymbol{v}_{j}\right|=\sqrt{1-c_{j, 0}^{2}}=\sqrt{\frac{n}{n+1}}$ 
and

$$
\boldsymbol{v}_{j} \cdot \boldsymbol{v}_{j^{\prime}}=-c_{j, 0} c_{j^{\prime}, 0}=-\frac{1}{n+1}
$$

for $j^{\prime} \neq j$. Hence, the angle between different vectors $v_{j}$ and $v_{j}$, is given by

$\theta_{j, j^{\prime}}=\theta_{n}=\arccos \left(-\frac{1}{n}\right)$

For the cases $n=1,2$ and 3 , this angle equals $\theta_{1}=\arccos (-1)=180^{\circ}, \theta_{2}=\arccos \left(-\frac{1}{2}\right)=120^{\circ}$ and $\theta_{3}=\arccos \left(-\frac{1}{3}\right) \approx 109.471^{\circ}$, respectively. These values correspond to the hybrid orbitals $\mathrm{sp}, \mathrm{sp}^{2}$ and $\mathrm{sp}^{3}$, respectively.

We also note that each real and normalized linear combination of the $\mathrm{p}_{x k}$ orbitals may be obtained by rotating $\mathrm{p}_{x 1}$. Hence, Equation 2 may be written as

$$
\psi_{j}=\frac{\mathrm{s}+\sqrt{n} \mathrm{p}_{\boldsymbol{v}_{j}}}{\sqrt{n+1}}
$$

where $\mathrm{p}_{v j}$ is the real $\mathrm{p}$ orbital along $v_{j}$. Since the s orbital is isotropic, all the $\mathrm{sp}^{n}$ orbitals, for a given $n$, have the same shape.

\section{References}

1. Pauling L. The nature of the chemical bond. Application of results obtained from the quantum mechanics and from a theory of paramagnetic susceptibility to the structure of molecules. Journal of the American Chemical Society. 1931; 53(4):13671400. http://dx.doi.org/10.1021/ja01355a027.

2. McMurry JE. Organic chemistry. Int. ed. Surrey: Ed. Brooks/ Cole; 2011.

3. Brown TE, LeMay HEH and Bursten BE. Chemistry: the central science. New Jersey: Prentice Hall; 1997.

4. Hsu CY and Orchin M. A simple Method for generating sets of orthonormal hybrid atomic orbitals. Journal of Chemical Education. 1973; 50(2):114. http://dx.doi.org/10.1021/ ed050p114.

5. Weinhold F and Brunck TK. The principle of maximum overlap. Journal of the American Chemical Society. 1976; 98(13):3745-3749. http://dx.doi.org/10.1021/ja00429a001.

6. Álvarez-Bajo O and Lemus R. General approach for the construction of hybrid orbitals. International Journal of Quantum Chemistry. 2006; 106(2):401-414. http://dx.doi. org/10.1002/qua.20755.

7. Marzari N, Mostofi AA, Yates JR, Souza I and Vanderbilt D. Maximally localized Wannier functions: Theory and applications. Reviews of Modern Physics. 2012; 84(4):14191475. http://dx.doi.org/10.1103/RevModPhys.84.1419.

8. Posternak M, Baldereschi A, Massidda S and Marzari N. Maximally localized Wannier functions in Antiferromagnetic $\mathrm{MnO}$ within the FLAPW Formalism. Physical Review B: Condensed Matter and Materials Physics. 2002; 65(18):184422. http://dx.doi.org/10.1103/PhysRevB.65.184422.

9. Hurley AC. On Orbital Theories of Molecular Structure. [Thesis]. Cambridge: Trinity College; 1952.

10. Boys SF. Construction of some molecular orbitals to be approximately invariant for changes from on molecule to

\section{Conclusions}

We have shown that the standard $\mathrm{sp}^{n}$ hybrid orbitals are the orthogonal linear combinations of one $\mathrm{s}$ and $n$ real $\mathrm{p}$ orbitals that minimize the total quadratic spread. This helps to understand why maximally-localized Wannier functions may resemble hybrid orbitals in crystalline materials ${ }^{7,8}$. It is the case when the set of energy bands under consideration is essentially reproduced by using a linear combination of atomic orbitals. In such cases, the multiband Wannier functions of maximal localization should mimic the atomic orbitals. Because of the requirement of maximal localization, such orbitals are hybrid orbitals instead of pure s or p orbitals. We also note that the maximally-localized orbitals are useful when the individual contribution of each constituent atom is under investigation ${ }^{15,16}$.

Finally, we would like to point out that it has been proposed that hybrid orbitals should better be excluded from the Chemistry curriculum ${ }^{17}$. Reinforcing several claims against such a proposal ${ }^{18,19}$, we stress that the growing importance of Wannier functions in Materials Science brings renewed interest on hybrid orbitals. Furthermore, we believe the present approach also applies to hybrid orbitals that include $\mathrm{d}$ and/or f states, such as the icosahedral ones ${ }^{20}$.

\section{Acknowledgements}

We thank the Brazilian Agencies CNPq and FAPESP for financial support.

another. Reviews of Modern Physics. 1960; 32(2):296-299. http://dx.doi.org/10.1103/RevModPhys.32.296.

11. Foster JM and Boys SF. Canonical configurational interaction procedure. Reviews of Modern Physics. 1960; 32(2):300-302. http://dx.doi.org/10.1103/RevModPhys.32.300.

12. Edmiston $\mathrm{C}$ and Ruedenberg K. Localized atomic and molecular orbitals. Reviews of Modern Physics. 1963; 35(3):457-464. http://dx.doi.org/10.1103/RevModPhys.35.457.

13. Edmiston $\mathrm{C}$ and Ruedenberg K. Localized atomic and molecular orbitals II. The Journal of Chemical Physics. 1965; 43(10):S97. http://dx.doi.org/10.1063/1.1701520.

14. Anton H. Calculus. 5th ed. New York: John Wiley \& Sons; 1995. v. 2.

15. Mayer I. Atomic orbitals from molecular wave functions: The effective minimal basis. Journal of Physical Chemistry. 1996; 100(15):6249-6257. http://dx.doi.org/10.1021/jp952779i.

16. Yao Y-X, Wang C-Z and Ho K-M. Chemical bonding analysis for solid-state systems using intrinsic oriented quasiatomic minimal-basis-set orbitals. Physical Review B: Condensed Matter and Materials Physics. 2010; 81(23):235119. http:// dx.doi.org/10.1103/PhysRevB.81.235119.

17. Grushow A. Is It Time To Retire the Hybrid Atomic Orbital? Journal of Chemical Education. 2011; 88(7):860-862. http:// dx.doi.org/10.1021/ed100155c.

18. Landis CR and Weinhold F. Comments on Is It Time To Retire the Hybrid Atomic Orbital? Journal of Chemical Education. 2012; 89(5):570-572. http://dx.doi.org/10.1021/ed200491q.

19. Truhlar DG. Are Molecular Orbitals Delocalized? Journal of Chemical Education. 2012; 89(5):573-574. http://dx.doi. org/10.1021/ed200565h.

20. Torres EM. Icosahedral hybrid orbitals. Chemical Physics Letters. 2000; 320(5-6):523-526. http://dx.doi.org/10.1016/ S0009-2614(00)00302-X. 